\title{
Influence of Plasma Treatment to the Performance of Amorphous IGZO based Flexible Thin Film Transistors
}

\author{
Long-long Chen, Xiang Sun, Ji-feng Shi, Xi-feng Li * Xing-wei Ding and Jian-hua Zhang * \\ Shanghai University, Key Laboratory of Advanced Display and System Applications of Ministry of \\ Education, 149 Yanchang Road, Shanghai, China; llchen@i.shu.edu.cn (L.C.); nivlaalvin@shu.edu.cn (X.S.); \\ shijf@shu.edu.cn (J.S.); dingxingwei@163.com (X.D.) \\ * Correspondence: lixifeng@shu.edu.cn (X.L.); jhzhang@shu.edu.cn (J.Z.); Tel.: +86-21-5633-4402 (X.L.)
}

\begin{abstract}
Thin film transistors (TFTs) using In-Ga-Zn Oxide (IGZO) as active layer and the gate insulator was treated with $\mathrm{NH}_{3}$ plasma and $\mathrm{N}_{2} \mathrm{O}$ plasma, respectively, which is fabricated on flexible PI substrate in this work. The performance of IGZO TFTs with different plasma species and treatment time are investigated and compared. The experiment results show that the plasma species and treatment time play an important role in the threshold voltage, field-effect mobility, Ion/Ioff ratio, sub-threshold swing (SS) and bias stress stability of the devices. The TFT with a 10 seconds NH3 plasma treatment shows the best performance; specifically, threshold voltage of $0.34 \mathrm{~V}$, field-effect mobility of $15.97 \mathrm{~cm}^{2} / \mathrm{Vs}$, Ion/Ioff ratio of $6.33 \times 10^{7}$, and sub-threshold swing of $0.36 \mathrm{~V} / \mathrm{dec}$. The proposed flexible IGZO-TFTs in this paper can be used as driving devices in the next-generation flexible displays.
\end{abstract}

Keywords: TFTs; IGZO; flexible; flasma treatment

\section{Introduction}

With the rapid development of AMOLED display technology, AMOLED display technology in compare to AMLCD display technology which exhibit more excellent properties, such as response faster, thinner, color is more brightly [1-3]. Glass substrate is the most commonly used in AMOLED and AMLCD display substrate manufacturing process, because of its high surface flatness, transparency, material stability, etc. At the same time, flexible display technology will be an important direction of display technology development now and in the future, but due to the hardness of large scale glass substrate which can't meet the requirements of flexible display substrate, the demand for flexible substrate technology were put forward. Plastic, metal film, ultra-thin glass, polyimide (PI) and other materials were used in flexible display research at present, and which can create a thinner, flexible display compared to traditional substrate technology [4-6]. At the same time, in order to develop the flexible display technology, traditional AMOLED display technology equipment and process technology were hoped used directly based on the traditional glass substrate fabrication process, except for low temperature process subject to special restrictions on flexible materials.

TFTs backplane active layer material technology progress past several years. Amorphous IGZO as a representative of oxide semiconductor TFT devices absorbed a lots of attention because of its higher field-effect mobility more than $10 \mathrm{~cm} 2 / \mathrm{Vs}$, lower of preparation process temperature, little affected by visible light and can be made into transparent devices, etc.[7-9]. So the IGZO based oxide semiconductor technology is optimum choice for flexible AMOLED display technology. However, according to the relevant reports [10-11], the electrical properties of the IGZO based TFTs devices characteristics, such as threshold voltage, sub-threshold swing (SS) and so on are affected by the process of film forming conditions. The threshold voltage is normally negative which can't meet the requirements of device drivers of AMOLED displays. Current of the IGZO based TFTs devices unable to be turned off or TFTs devices will be opened at low voltage are technical issues. 
In this latest work, the IGZO based TFTs devices with silicon nitride and silicon oxide layers stacked as gate insulator and $\mathrm{NH}_{3}$ plasma surface treatment is demonstrated for the first time. In addition, $\mathrm{N}_{2} \mathrm{O}$ plasma surface treatment is also used to distinguish the impacts of characteristic of the IGZO TFTs device (cf. Figure 1).

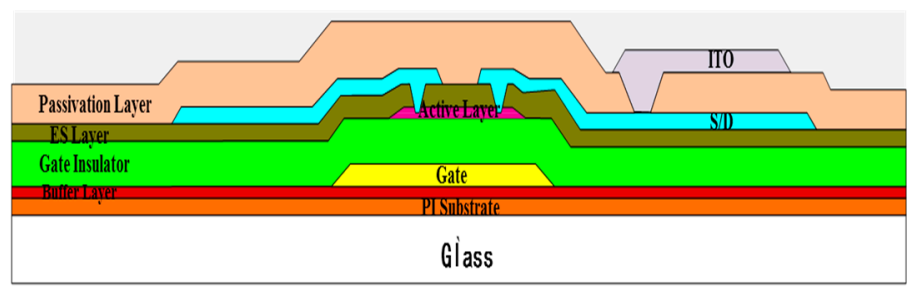

Figure 1. Schematic drawing of the flexible TFTs structures.

\section{Materials and Methods}

The fabrication of devices started by coating a 15-um PI flexible substrate on TFT glass substrate and baked for an hour at $220^{\circ} \mathrm{C}$. Then, a 150-nm silicon nitride was deposited by plasma-enhanced chemical vapor deposition (PECVD) at $220^{\circ} \mathrm{C}$ capped with a 150-nm silicon oxide which deposited with the same temperature continuously as a buffer layer for flexible substrate. The buffer layer on one hand improved adhesion of deposited TFTs films, on the other hand, sequestered water vapor, oxygen effect of TFTs devices on the flexible substrate. TFTs device cross-sectional view of bottom gate TFT structure is as shown in Figure 1. Then, a 100-nm molybdenum and a 50-nm ITO were sputtered continually, patterned and wet etched for gate electrode. ITO layer used here for the dry etching stop layer to molybdenum which the dry etching selection ratio is about 1:3 of molybdenum and silicon oxide. Then, a 250-nm silicon nitride and 50-nm silicon oxide were deposited by PECVD at $220^{\circ} \mathrm{C}$ for device gate insulator layer in order to increase the dielectric constant value. Then, gate insulator surface was treated 10 seconds by $\mathrm{N}_{2} \mathrm{O}$ plasma, 10 seconds by $\mathrm{NH}_{3}$ plasma, 120 seconds by $\mathrm{NH}_{3}$ plasma, respectively. Controlled TFTs device is prepared as contrast. Then, a 50-nm amorphous IGZO was sputtered at room temperature, the sputtering power was at $300 \mathrm{~W}$ and gas flow of Ar and $\mathrm{O} 2$ were $50 \mathrm{sccm}$ and $5 \mathrm{sccm}$. The active layer was patterned and etching for active island structure. Then a 200-nm silicon oxide was deposited by PEDVD at $250^{\circ} \mathrm{C}$ as etching stop layer. It was patterned as via hole for the electrical contact between the active layer and source/ drain electrodes. The technology process of source and drain electrodes were sputtered and patterned using the same process as gate layer. Then a 200-nm silicon oxide was deposited as passivation layer. Dry etching was employed to form contact hole for electrical contact between pixel and drain. Finally, a 50-nm ITO film was sputtered at room temperature and patterned, etched as pixel patterns. In order to improve TFTs device stability, the substrate was annealed in atmosphere at $200{ }^{\circ} \mathrm{C}$ of 2 hours. The active layer channel of width (W)/ length (L) of TFTs TEG is $6 \mathrm{um} / 10 \mathrm{um}$. Figure 2 shows the top view of panel on the flexible PI substrate and TFTs devices to be measured, respectively.

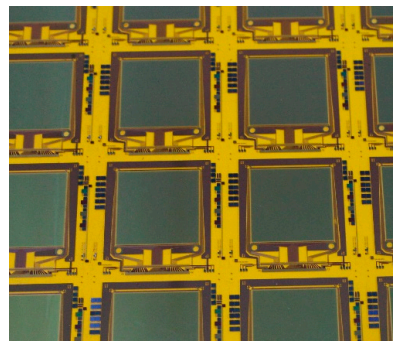

(a)

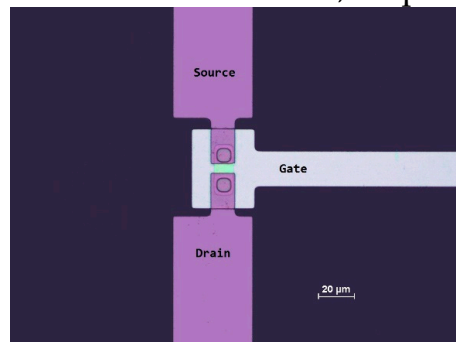

(b)

Figure 2. (a) Photo of panel manufactured on flexible PI substrate, TFTs TEG on the left of each shot of panel (b) Top view of TFTs TEG which the active layer channel of $W / L$ is $6 \mathrm{um} / 10 \mathrm{um}$.

The fabricated IGZO-TFTs without plasma treatment were named NO. 1, with $\mathrm{N}_{2} \mathrm{O}$ plasma treatment of 10 seconds were named NO. 2, and with $\mathrm{NH}_{3}$ plasma treatment of 10 / 120 seconds were 
named NO. 3 / 4, respectively. Research manuscripts reporting large datasets that are deposited in a publicly available database should specify where the data have been deposited and provide the relevant accession numbers. If the accession numbers have not yet been obtained at the time of submission, please state that they will be provided during review. They must be provided prior to publication.

\section{Results}

The transfer characteristic curves of IGZO based flexible TFTs under various atmosphere treatment species and times were shown in Figure 3. Threshold voltage presenting an optimistic positive shift with the plasma treatment. It shown that the increased threshold voltage could be ascribed to the decrease of carrier density, the feature could be fit for most of the traditional display driver circuits. The Ion/Ioff ratio of samples were measured at approximately $2.41 \times 10^{7}, 2.46 \times 10^{7}$, $6.33 \times 10^{7}$ and $6.10 \times 10^{6}$ for sample NO. 1 to 4 .

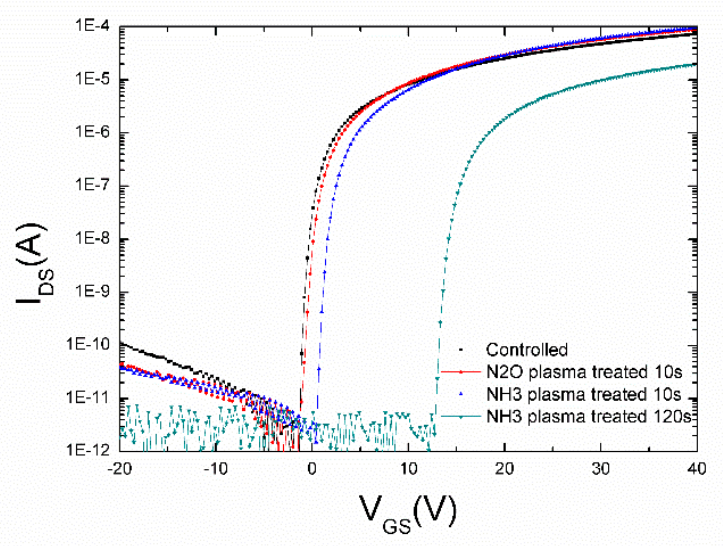

Figure 3. Transfer characteristic curves of IGZO based flexible TFTs after various treatment.

From the $I_{D S}^{1 / 2}$-VGS curves shown in Figure 4, field-effect mobility $(\mu)$ and threshold voltage $\left(V_{\mathrm{TH}}\right)$ can be extracted according to the following expression:

$$
I_{D S}=\frac{w}{2 L} \mu C_{i}\left(V_{G S}-V_{T H}\right)^{2} \quad V_{D S}>V_{G S}-V_{T H}
$$

Where $C_{i}$ is the capacitance per unit of the gate insulator layer of $15.4 \mathrm{nF} / \mathrm{cm}^{2} . W$ and $L$ are active layer channel width and length, $V_{\mathrm{DS}}$ and $V_{\mathrm{GS}}$ are source-drain voltage and gate-substrate voltage, respectively. The threshold voltage and field-effect mobility are of $-4.42 \mathrm{~V}$ and $8.97 \mathrm{~cm}^{2} / \mathrm{Vs},-2.39 \mathrm{~V}$ and $12.49 \mathrm{~cm}^{2} / \mathrm{Vs}, 0.34 \mathrm{~V}$ and $15.97 \mathrm{~cm}^{2} / \mathrm{Vs}, 10.58 \mathrm{~V}$ and $5.58 \mathrm{~cm}^{2} / \mathrm{Vs}$ of the sample NO. 1 to 4 , respectively.

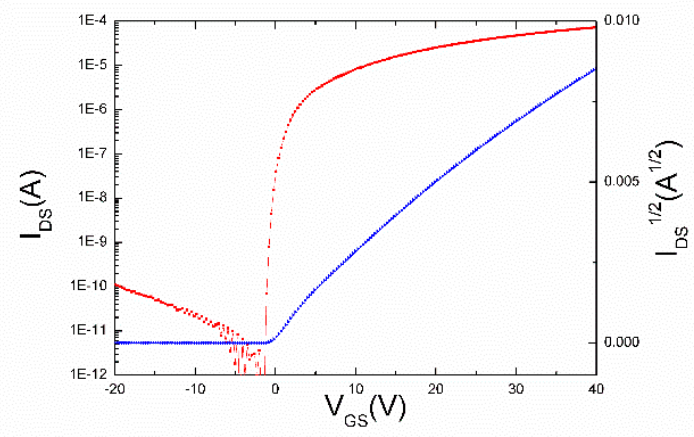

(a)

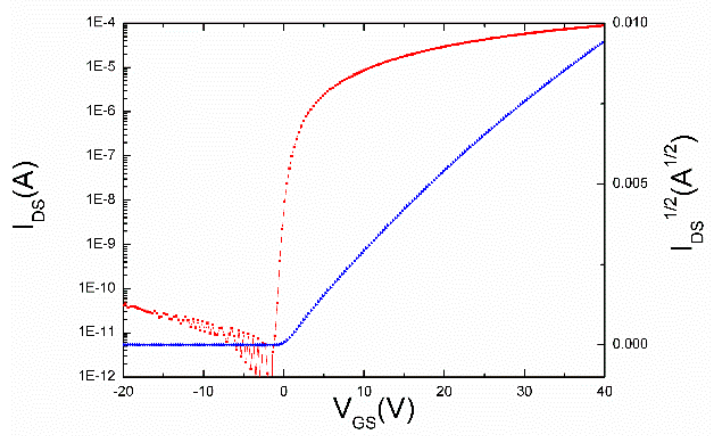

(b) 


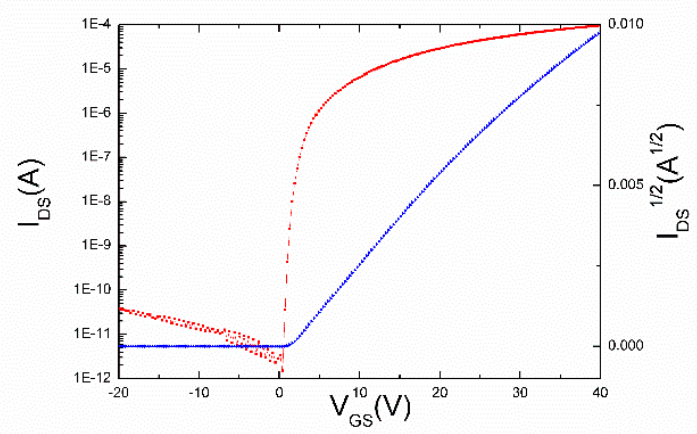

(c)

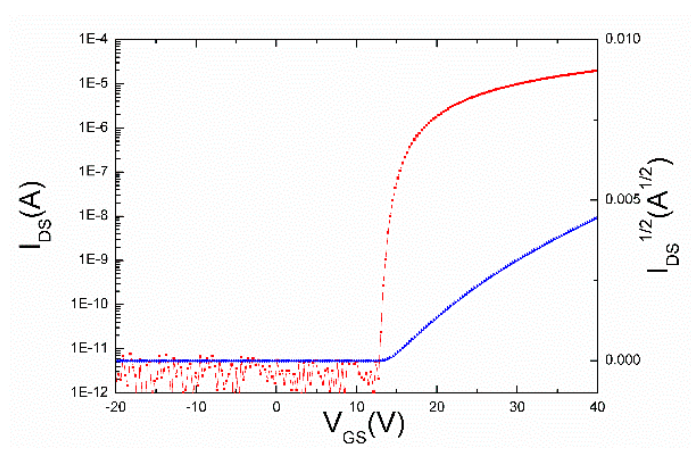

(d)

Figure 4. Corresponding transfer characteristic IDS versus $\mathrm{V}_{\mathrm{GS}}$ at a fixed $\mathrm{V}_{\mathrm{DS}}=10.1 \mathrm{~V}$ and the $\mathrm{I}_{\mathrm{DS}}^{1 / 2}-\mathrm{V}_{\mathrm{GS}}$ curves of TFTs with various treatments. (a) Sample NO. 1. (b) Sample NO. 2. (c) Sample NO. 3. (d) Sample NO. 4.

Sub-threshold swing (SS) of samples can be calculated from the formula:

$$
\mathrm{SS}=\frac{d V_{G S}}{d\left(\log I_{D S}\right)},
$$

Here we measured the value of SS of $0.47 \mathrm{~V} / \mathrm{dec}, 0.39 \mathrm{~V} / \mathrm{dec}, 0.36 \mathrm{~V} / \mathrm{dec}, 0.45 \mathrm{~V} / \mathrm{dec}$, respectively. Additional are listed in Table 1.

Table 1. Electrical characteristic of various treated TFTs

\begin{tabular}{cccccc}
\hline Samples & No. & $\boldsymbol{V}_{\mathrm{TH}}(\mathbf{V})$ & $S S(\mathbf{V} / \mathbf{D e c})$ & $\mu\left(\mathbf{c m}^{2} / \mathbf{V s}\right)$ & $\mathbf{I}_{\text {on }} / \mathbf{I}_{\text {off }}$ \\
\hline Controlled & 1 & -4.42 & 0.47 & 8.97 & $2.41 \times 10^{7}$ \\
$\mathrm{~N}_{2} \mathrm{O}$ treatment 10s & 2 & -2.39 & 0.39 & 12.49 & $2.46 \times 10^{7}$ \\
$\mathrm{NH}_{3}$ treatment 10s & 3 & 0.34 & 0.36 & 15.97 & $6.33 \times 10^{7}$ \\
$\mathrm{NH}_{3}$ treatment 120s & 4 & 10.58 & 0.45 & 5.58 & $6.10 \times 10^{6}$ \\
\hline
\end{tabular}

As shown in Figure 5(a), the threshold voltage $V_{\mathrm{TH}}$ increased with the plasma treatment to the TFTs gate insulators. The $V_{\text {TH }}$ of $0.34 \mathrm{~V}$ was obtained as the gate insulator treated by $\mathrm{NH}_{3}$ plasma of 10 seconds, and increase to $10.58 \mathrm{~V}$ with treatment of 120 seconds. The IGZO-TFT with $\mathrm{N}_{2} \mathrm{O}$ plasma treatment of 10 seconds shows a $V_{\text {TH }}$ of $-2.39 \mathrm{~V}$.

As shown in Figure 5(b), sample NO. 3 shows a smallest SS value of $0.36 \mathrm{~V} / \mathrm{dec}$, which indicate that $\mathrm{NH}_{3}$ plasma treatment has a positive effect on decreasing the trappings at the gate insulator/active layer interface. Sample NO. 4 with the $\mathrm{NH}_{3}$ plasma treatment of 120 seconds shows a field-effect mobility of $5.58 \mathrm{~cm}^{2} / \mathrm{Vs}$ and a sub-threshold swing of $0.45 \mathrm{~V} / \mathrm{dec}$, indicating that the surface interface may be destroyed by the long times of ion bombardment. We concluded that the 10 seconds $\mathrm{NH}_{3}$ plasma treatment is the optimal condition.

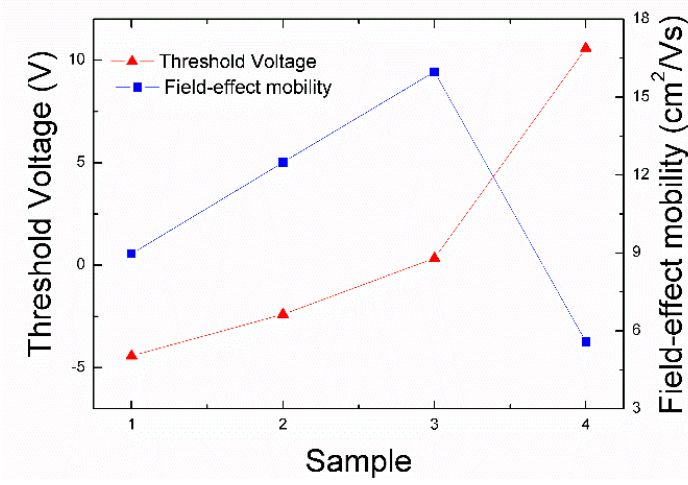

(a)

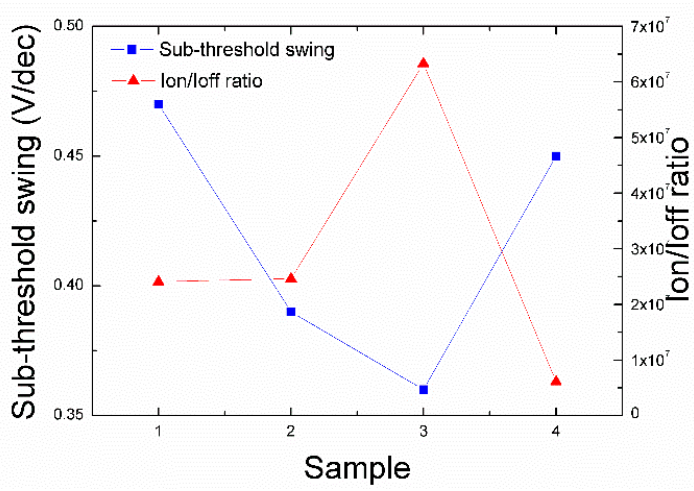

(b)

Figure 5. (a) threshold voltage and field-effect mobility of samples NO. 1 to 4 . (b) sub-threshold voltage and Ion/Ioff ratio of samples NO. 1 to 4 . 

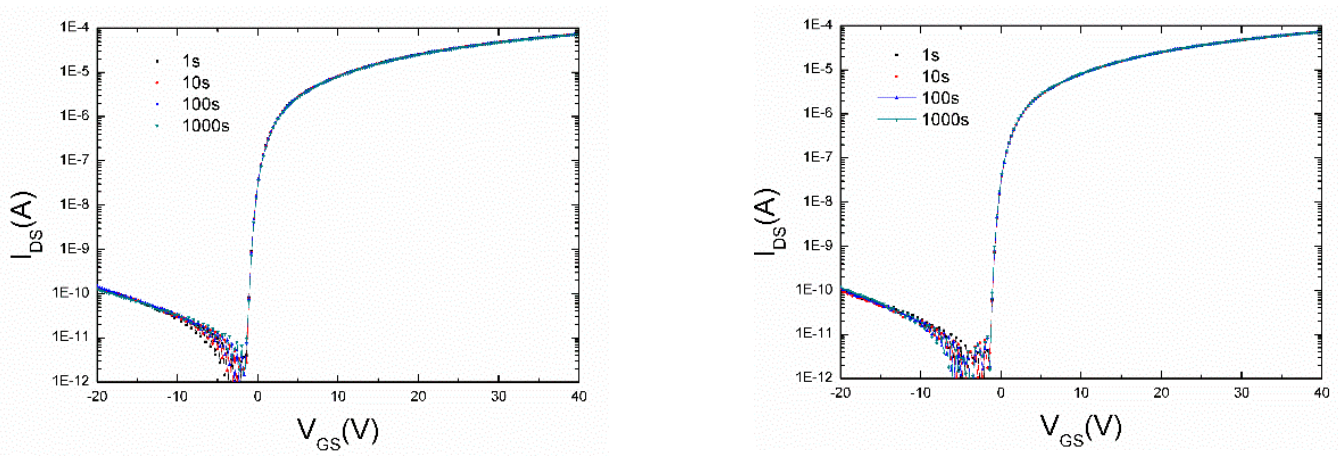

(a)
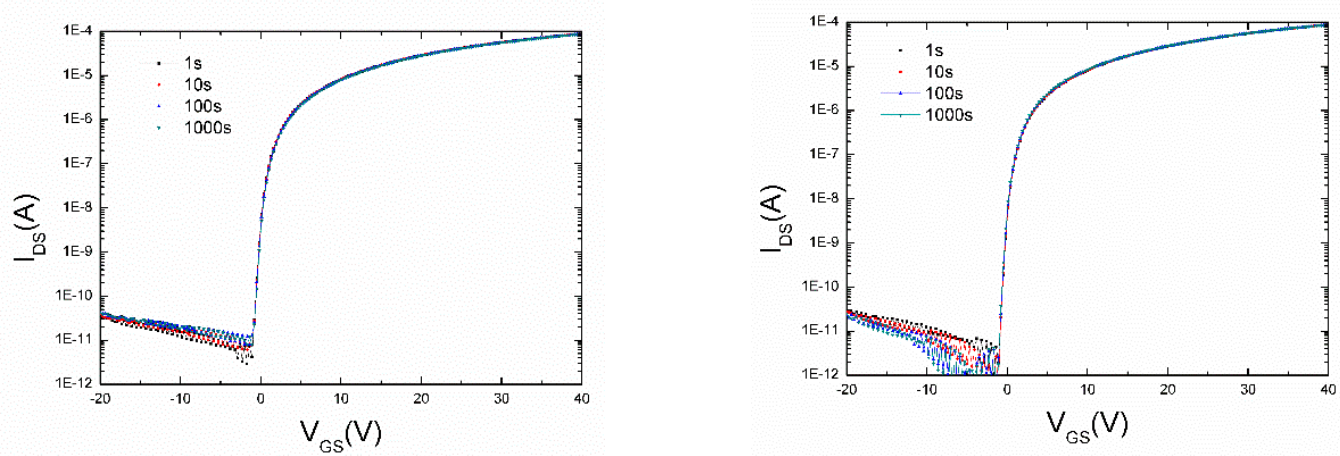

(b)
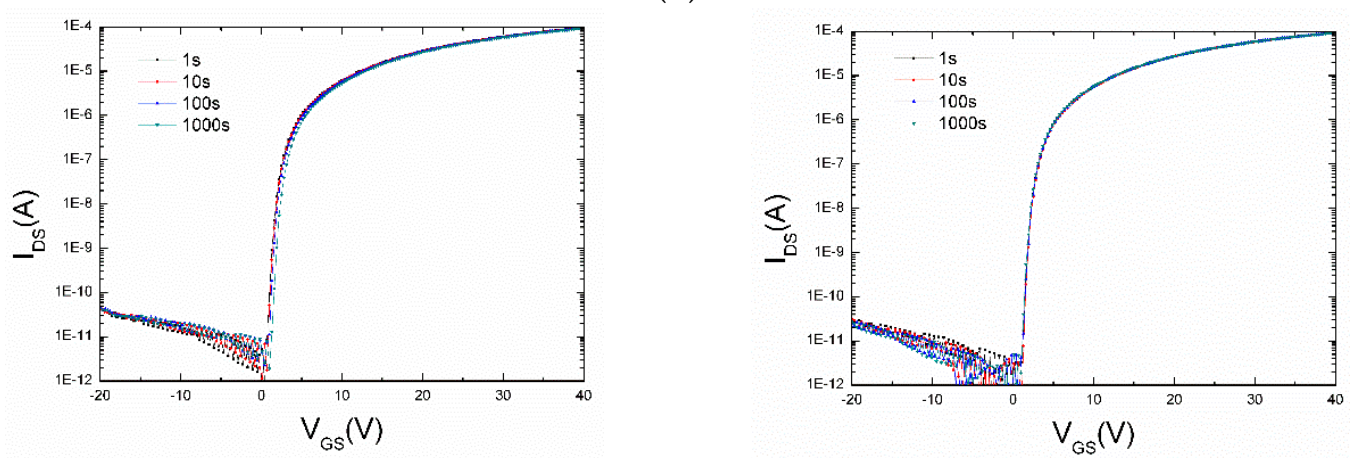

(c)
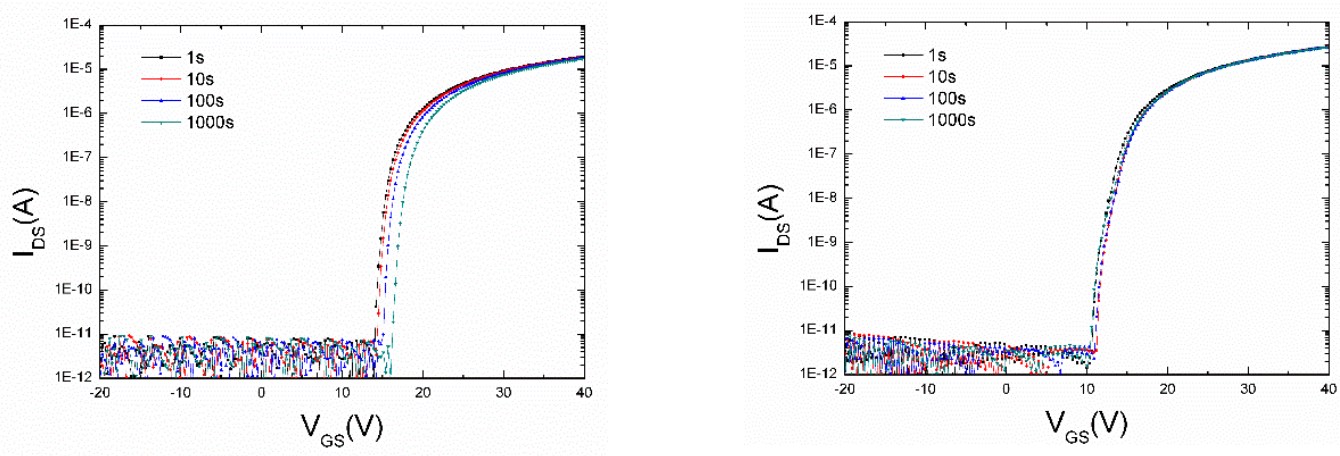

(d)

Figure 6. The transfer characteristic curves of IGZO based flexible TFTs. The gate bias voltage of $20 \mathrm{~V}$ at PBST and which of $-20 \mathrm{~V}$ at NBST for 1000 seconds at room temperature, respectively. (a) PBST and NBST: No. 1. (b) PBST and NBST: No. 2. (c) PBST and NBST: No. 3. (d) PBST and NBST: No. 4.

Positive bias stress test (PBST) and negative bias voltage stress test (NBST) were carried out to further investigate the effect of plasma treatment on the stability of TFTs as shown in Figure 6. The gate bias voltage of 20 or $-20 \mathrm{~V}$ was applied for 1000 seconds at room temperature in atmosphere. VGS loaded on the TFTs continuously during the voltage stress process, which is two or three times more 
than typical driving voltage for display panels. The threshold voltage shifts under bias voltage stress is attributed to electron trapping between interface of the active layer and gate insulator. The TFT with a 10 seconds $\mathrm{NH}_{3}$ plasma treatment also shows excellent stability under bias voltage stress. The threshold voltage shift were $0.12 \mathrm{~V}$ and $0.26 \mathrm{~V}$ after a $20 \mathrm{~V}$ and a $-20 \mathrm{~V}$ gate voltage stressed for 1000 seconds, while the value for the sample NO. 1, 2 and 4 are $-0.44 \mathrm{~V}$ and $-0.92 \mathrm{~V},-2.03 \mathrm{~V}$ and $-1.16 \mathrm{~V}$, $4.56 \mathrm{~V}$ and $0.14 \mathrm{~V}$, respectively.

\section{Conclusions}

In conclusion, TFTs using IGZO as active layer and the gate insulator was treated with plasma, which are fabricated on flexible PI substrate in this work. TFTs treated by $\mathrm{NH}_{3}$ plasma of 10 seconds shows the best performance than other samples under various treatment conditions, such as threshold voltage of $0.34 \mathrm{~V}$, field-effect mobility of $15.97 \mathrm{~cm}^{2} / \mathrm{Vs}$, Ion/ Ioff ratio of $6.33 \times 10^{7}$, and subthreshold swing of $0.36 \mathrm{~V} / \mathrm{dec}$. The TFT with a 10 seconds $\mathrm{NH}_{3}$ plasma treatment also shows stability under bias voltage stress test, the threshold voltage shift were $0.12 \mathrm{~V}$ and $0.26 \mathrm{~V}$ after a $20 \mathrm{~V}$ and a $20 \mathrm{~V}$ gate voltage stressed for 1000 seconds. Therefore, the $\mathrm{NH}_{3}$ plasma treatment 10 seconds on the gate insulator of IGZO based flexible TFTs is an appropriately solution for improving the TFTs performance nowadays.

Acknowledgments: This work is supported by the project of Science and Technology Commission of Shanghai Municipality (Grant No. 16JC1400602), National Natural Science Foundation of China (Grant No. 61006005), Shanghai Sailing Program (17YF1406300), and Project of National Post-Doctor Fund (2015M580315).

Author Contributions: C.L. and Z.J. conceived and designed the experiments; S.X. and S.J performed the experiments; L.X. and Z.J. analyzed the data; D.X. contributed reagents/materials/analysis tools; C.L. wrote the paper.

Conflicts of Interest: The authors declare no conflict of interest.

\section{References}

1. Wu, C.C.; Theiss, S.D.; Gu, G.; Lu, M.H. Integration of Organic LEDs and Amorphous Si TFTs onto Flexible and Lightweight Metal Foil Substrates. IEEE Electron Device Lett. 1997, 18, 609-612.[CrossRef]

2. Zhou, L.S.; Wanga, A.; Wu, S.C. All-organic active matrix flexible display. Appl. Phys. Lett. 2006, 88, 083502.

3. Jeong, J.K.; Jin, D.U.; Shin, H.S.; Lee, H.J. Flexible Full-Color AMOLED on Ultrathin Metal Foil. IEEE Electron Device Lett. 2007, 28, 389-391. [CrossRef]

4. Plichta, A.; Weber, A. Ultra-thin flexible glass substrates. Proc. Mater. Res. Soc. Symp. 2003, 769, H9.1H9.10. [CrossRef]

5. Cheng, I.C.; Kattamis, A.; Long, K.; Sturm, J.C.; Wagner, S. Stress control for overlay registration in a-Si:H TFTs on flexible organic polymer-foil substrates. J. Soc. Inf. Display, 2005, 13, 563-568. [CrossRef]

6. Choi, M.H.; Kim, B.S.; Jang, J. High-Performance Flexible TFT Circuits Using TIPS Pentacene and Polymer Blend on Plastic. IEEE Electron Device Lett. 2012, 33, 1571-1573. [CrossRef]

7. Lim, W.; Jang, J.H.; Kim, S.H.; Norton, D.P.; Craciun, V.; Pearton, S.J.; Ren, F.; Shen, H. High performance indium gallium zinc oxide thin film transistors fabricated on polyethylene terephthalate substrates. Appl. Phys. Lett. 2008, 93, 082102. [CrossRef]

8. Chen, C.; Abe, K; Kumomi, H.; Kanicki, J. Density of States of a-InGaZnO from Temperature-Dependent Field-Effect Studies. IEEE Trans. Electron Devices, 2009, 56, 1177-1183. [CrossRef]

9. Hsu, H.H.; Chang, C.Y.; Cheng, C.H.; Yu, S.H.; Su, C.Y.; Su, C.Y. Fully room-temperature IGZO thin film transistors adopting stacked gate dielectrics on flexible polycarbonate substrate. Solid-State Electron. 2013, 89, 194-197. [CrossRef]

10. Liu, C.; Wei, M.; Yang, F.; Jia, Z.; Deng, H. Effects of Electrode Materials on The Performances of IGZObased Thin Film Transistor. Chin. J. of Lumin. 2014, 35, 1365 1369. [CrossRef]

11. Jia, T.Y.; Zhan, R.Z.; Dong, C.Y. Simulation of the Stability of a-IGZO TFT-OLED Pixel Circuits. Chin. J. of Lumin. 2013, 34, 1240 1244. [CrossRef]

(C) 2017 by the authors. Licensee Preprints, Basel, Switzerland. This article is an open access article distributed under the terms and conditions of the Creative Commons by Attribution (CC-BY) license (http://creativecommons.org/licenses/by/4.0/). 